\title{
TURISMO EN LOS CEMENTERIOS. EL CASO DE LA NECRÓPOLIS DE COLÓN, LA HABANA, CUBA
}

\section{TOURISM AT THE CEMETERIES. WALKING AMONG THE DEAD}

\author{
Eros Salinas-Chávez ${ }^{1}$ \\ Jeisy Diaz-Fernández ${ }^{2}$ \\ Universidad de La Habana, Cuba
}

\begin{abstract}
RESUMEN
Los cementerios se han convertido hoy en día, en verdaderos museos al aire libre, que revelan un gran interés para las visitas. Este hecho no es la expresión de sentimientos morbosos o esnobistas, sino el innegable deseo de conocer mejor la historia y la cultura de los países y ciudades visitadas. Son numerosos los cementerios en el mundo, en los que se han diseñado recorridos turísticos, que se comercializan preferentemente como ofertas de excursiones. Este artículo tiene como objetivo destacar el actual uso turístico de los cementerios y la necesidad de planificar sus capacidades de carga turística tomando en consideración distintas variables y algunos criterios para la interpretación de este patrimonio. Todo ello se ejemplifica en el caso del cementerio de Cristóbal Colón en La Habana: se caracteriza la instalación, se muestra una síntesis de sus valores patrimoniales y se evalúan los límites de uso con fines turísticos, que permitan percibir una adecuada experiencia a los visitantes y faciliten la conservación de ese valioso patrimonio y se mantengan las funciones necrológicas de estos sitios.
\end{abstract}

Palabras clave: cementerios, patrimonio funerario, necroturismo, capacidad de carga turística.

1 Doctor en Ciencias Geográficas. Investigador Titular del Instituto de Geografía Tropical. Profesor Titular de la Facultad de Turismo de la Universidad de La Habana. Correo electrónico: esalinas@ftur.uh.cu

2 Master en Gestión Turística. Profesora de la Facultad de Turismo de la Universidad de La Habana. Correo electrónico: jeisy_diaz@ftur.uh.cu 
Eros Salinas-Chávez, Jeisy Diaz-Fernández. Turismo en los cementerios.

El caso de la Necrópolis de Colón, La Habana, Cuba

\begin{abstract}
Today, cemeteries are becoming true open-air museums that reveal a great interest for visitors. This is not a morbid expression, but the undeniable desire to know more about the history and culture of countries and cities visited. There are numerous cemeteries in the world where tours are designed and marketed mainly as excursions. This article aims at highlighting the existing use of cemeteries for tourism and the need to plan the tourist capacity taking into account different variables and some criteria applied for the heritage interpretation. The magnificent Cristobal Bolon Necropolis of Havana is used as a case study, presenting the main aspects which characterize this graveyard. A briefing about its cultural values is presented and the boundaries for touristic product development is assessed, which will allow tourists to perceive an adequate experience as well as facilitate the preservation of this valuable cultural heritage, thus resulting in an achievement of successful coexistence of both tourism services and obituary functions at the place.
\end{abstract}

Keywords: cemeteries, funeral heritage, necrotourism, tourist capacity.

\title{
Introducción
}

Los cementerios, lugar de reposo de seres que fueron y que ya no están, se han convertido en la actualidad en verdaderos museos al aire libre, revelan un gran interés para las visitas y recorridos en muchas partes del mundo. Este hecho no es la expresión de sentimientos morbosos o esnobistas, sino el innegable deseo de conocer mejor la historia y la cultura de los países y ciudades visitadas. En cada ciudad existe algún cementerio digno de visitar, ya sea por sus ilustres huéspedes, sus valores históricos o por la belleza de su arquitectura o mejor aún por todas esas razones combinadas.

Los cementerios, son el espejo donde se contemplan las raíces más profundas de los pueblos, la palabra cementerio significa lugar de descanso y comprende sitios descubiertos, por lo general cercados y ubicados en el interior del espacio de las ciudades y pueblos o en su entorno inmediato.

Un cementerio es algo más que un sitio donde yacen un conjunto de personas sepultadas, sea mucha o poca su importancia y valía, está presente de modo inobjetable la omnipresencia del tiempo, la maravillosa obra humana convertida en piedra y levantada como un elocuente y magnifico monumento diverso y multiforme, capaz de inducir a la reflexión colectiva y al conocimiento (Vento, 2002).

Son construcciones realizadas en una época siguiendo determinadas regulaciones sanitarias, normas y dictados arquitectónicos y estéticos, si son capaces de sostener su funcionabilidad, se convierten en instalaciones fuera del tiempo, donde pueden apreciarse los cambios de expresiones y valores sociales, políticos y culturales, que sobrepasan con creces las 
variaciones perceptibles a simple vista, de estilos arquitectónicos y urbanísticos para convertirse en lugares reflejo de las transformaciones de la vida pública de la sociedad.

Las necrópolis tienen la rara y excepcional cualidad de reunir en obligada concordia a sujetos en un tiempo enemigos, pero ahora igualados por un destino común imposible de evadir, los visitantes pueden tener conciencia de esta impronta en el tiempo detenido, y de ese aire de sutil distanciamiento con todo lo inmediato, excepto la muerte misma.

Estos sitios, monumentos, conjuntos y elementos funerarios constituyen un caso particular, poco valorado y menos atendido del patrimonio cultural material acompañado a su vez por usos, costumbres, ritos, conductas y manifestaciones de cada cultura en torno a la inevitabilidad de la muerte y la disposición de los restos humanos, lo cual constituye un patrimonio cultural inmaterial de igual importancia y una de las manifestaciones de la diversidad cultural que han acompañado al género humano desde tiempos muy remotos y seguirán acompañándolo hasta su extinción (Red Chilena de Cementerios Patrimoniales, 2006).

En los últimos años, diferentes autores han investigado sobre el uso de cementerios para el desarrollo turístico (Aruca y Salinas, 1992; Chepe, 1997; Richard, 2006; Aulet, 2010; Iznájar, 2013). En este sentido, se ha apuntado que los cementerios reúnen diferentes valores que los hacen ser espacios de fuerte atractivo cultural (Vento, op. cit.;Manzanarez, 2012; Aloy, 2013). Esta situación ha conllevado a que el Consejo de Europa, en el año 2010, reconociera como "itinerario cultural" a la Ruta Europea de los Cementerios (Martínez, 2014), que comprende el Camino de Santiago, La Vía Francigena, la Ruta de los Fenicios o del Legado Andalusí, que van conformando "recorridos por 63 camposantos localizados en 50 ciudades de 20 países europeos" (Benayas y Muñoz, 2007). También, han surgido los términos de "Dark Tourism" (Lennon y Foley, 2002; Stone y Sharpley, 2008) y Tanaturismo (Seaton, 2002; Jaffari, 2002) como una modalidad turística a raíz de toda la diversificación de esta oferta cultural la cual, según la web de la Compañía Gestora de los Camposantos Catalanes, tiene como "...objetivo... mostrar estos recintos no como lugares de muerte, sino de vida; ya que los cementerios son el resumen de nuestra historia más reciente y donde podremos encontrar muchas de nuestras referencias" (Manzanarez, 2012). 
El problema de la presente investigación es el siguiente: ¿Cómo compatibilizar el uso turístico de los cementerios con la conservación de sus valores patrimoniales y la utilización que de estos espacios hace la población local? A partir de lo anterior, se proponen como objetivos: realizar un diagnóstico general de los valores patrimoniales de los cementerios estudiados para su uso turístico; establecer un grupo de parámetros que permitan calcular las capacidades turísticas de los mismos; y proponer recomendaciones para mejorar la experiencia cognoscitiva y emocional que estos sitios provocan en los visitantes. Además, se realizó un diagnóstico sobre la situación actual que enfrenta el Cementerio de Colón de La Habana, en cuanto a la planificación interpretativa a partir de la metodología propuesta por Morales (2001).

En el procedimiento de investigación utilizado se destaca como herramientas de trabajo: la observación participativa derivada de los múltiples recorridos realizados por los cementerios cubanos, en particular por la Necrópolis de Colón, las entrevistas abiertas a guías turísticos especializados en estos lugares, consultas a especialistas de las entidades relacionadas con la protección del patrimonio cultural y al personal administrativo de los servicios necrológicos, dentro y fuera de los cementerios y la revisión bibliográfica de los programas de visitas existentes en Cuba y otros países del mundo.

\section{Algunos cementerios renombrados de interés turístico en el mundo}

Entre los cementerios más conocidos y visitados del mundo se encuentra, en París, en lo alto de una colina, el famoso cementerio Pére La Chaise, que ya existía en el Medioevo, donde yacen los restos de renombrados artistas y literatos con Alejandro Dumas, Oscar Wilde, Honoré de Balzac, Moliere, Federico Chopin, Víctor Hugo y Marcel Proust, entre otros.

Se distingue, entre otros, el Cementerio Judío de Praga, original por la carencia de lujosos panteones y monumentos de costosos mármoles, y por la presencia de miles de lápidas amontonadas en un reducido espacio que contienen el enterramiento de más de cien mil judíos; el cementerio de Highgate en Londres, donde está enterrado Carlos Marx y que está rodeado por la famosa leyenda del vampiro que merodea el camposanto; el cementerio de Staglieno en Génova, Italia, famoso por el valor artístico de sus esculturas funerarias ${ }^{2}$; el Laurel Hill, en Filadelfia, uno de los pocos de Estados Unidos que forman parte del patrimonio Histórico Nacional 
y el cementerio nacional de Arlington, de carácter militar, en el cual se encuentran los fallecidos de todas la contiendas bélicas desde la Guerra de Secesión y el panteón de la familia Kennedy.

Los cementerios de Poblenouy Montjuic en Barcelona, y La Almudena en Madrid. El cementerio Zentralfiedhof de Viena es el de los amantes de música clásica; yacen en él Ludwig van Beethoven, Johannes Brahms, Franz Schubert y Johan Strauss, entre otros destacados músicos. En Estocolmo, el cementerio del Bosque declarado Patrimonio Cultural de la Humanidad por la UNESCO, en 1994; se reconoce por su adaptación espacial a las características del relieve y la vegetación. En Buenos Aires, se encuentran dos importantes cementerios por sus valores patrimoniales: La Recoleta y La Chacarita, el primero famoso por la tumba de Eva Perón y Domingo Sarmiento; y el segundo por la Tumba de Carlos Gardel.

La importancia de los cementerios, desde el punto de vista de sus visitas turísticas, está asociada por lo general, con las personalidades cuyos restos allí se encuentran, por los significativos valores patrimoniales de los monumentos artísticos funerarios existentes (arquitectónicos, escultóricos y paisajísticos) y, además, por las nobles tradiciones, leyendas o simples curiosidades que de ellas son fieles depositarios. La trascendencia de estos recintos se asocia con la consolidación de la identidad cultural del grupo social, donde el patrimonio adquirirá valor en función de su capacidad como elemento de identificación y apropiación del entorno inmediato y del paisaje por parte de la comunidad (Perelló, 2005).

En noviembre del 2000, se realizó en Medellín, Colombia, el Primer Encuentro Andino de Valoración y Gestión de Cementerios Patrimoniales, el cual buscó crear un espacio de reflexión y análisis en torno a mecanismos de apropiación, gestión y recuperación de cementerios. Fruto de este encuentro, se creó la Red Latinoamericana de Cementerios Patrimoniales, integrada por un grupo de gestores que buscaban el reconocimiento, difusión, valoración, preservación y apropiación social del patrimonio cultural material, relativo a sitios, monumentos, conjuntos y elementos de carácter funerario, así como del patrimonio inmaterial asociado a los usos, costumbres y manifestaciones culturales que las comunidades expresan y representan en torno a la muerte.

En octubre del 2015, se celebró el II Taller sobre Cementerios y Espacios Funerarios de La Habana, convocado por el Grupo Gestor de 
Museología y Patrimonio de la Necrópolis de Colón, el mismo devino en excelente momento de intercambio y debate sobre temas relacionados con la conservación y uso de estos espacios, en particular de la relación entre cultura funeraria, turismo y comunidad.

\section{Cementerios cubanos: algo de historia}

Muchas son las construcciones cementeriales cubanas que poseen altos valores patrimoniales; ya sea por las expresiones de arte funerario que atesoran sus bóvedas y panteones, o por las personalidades que yacen en sus recintos.

Tradicionalmente, y tal vez con la creencia de estar "más cerca de Dios", las iglesias acogieron los enterramientos en casi todas las ciudades del mundo, lo que constituía, en cierta medida, un permanente atentado a las más elementales normas epidemiológicas; en Cuba, esta práctica cesó entre 1799-1812. En La Habana, correspondió a la iniciativa de José Díaz de Espada, más conocido como Obispo Espada y del sabio cubano y médico Tomás Romay, que el 2 de febrero de 1806 se inaugurara el primer cementerio público, conocido como Cementerio Espada.

Entre los recintos funerarios existentes en Cuba, importantes para ser visitados con una óptica turística, se destacan tres por sus valores patrimoniales, el cementerio de Santa Ifigenia, inaugurado en 1868, en Santiago de Cuba y declarado Monumento Nacional en 1937, pues atesora gran parte de la historia del país, a partir de las inhumaciones de relevantes próceres de la independencia, entre ellos Carlos Manuel de Céspedes, el Padre de la Patria, y el mausoleo al Apóstol José Martí, Héroe Nacional, inaugurado el 30 de junio de 1951. El cementerio Tomás Acea, en Cienfuegos -inaugurado en 1926-, modelo único en Cuba de cementerio jardín, atravesado por amplias avenidas flanqueadas por árboles de diferentes especies frutales y ornamentales (figuras 1 y 2).El tercer cementerio es el Cristóbal Colón, en La Habana (figuras 3 y 4). 
Figura 1. Cementerio Santa Ifigenia. Santiago de Cuba

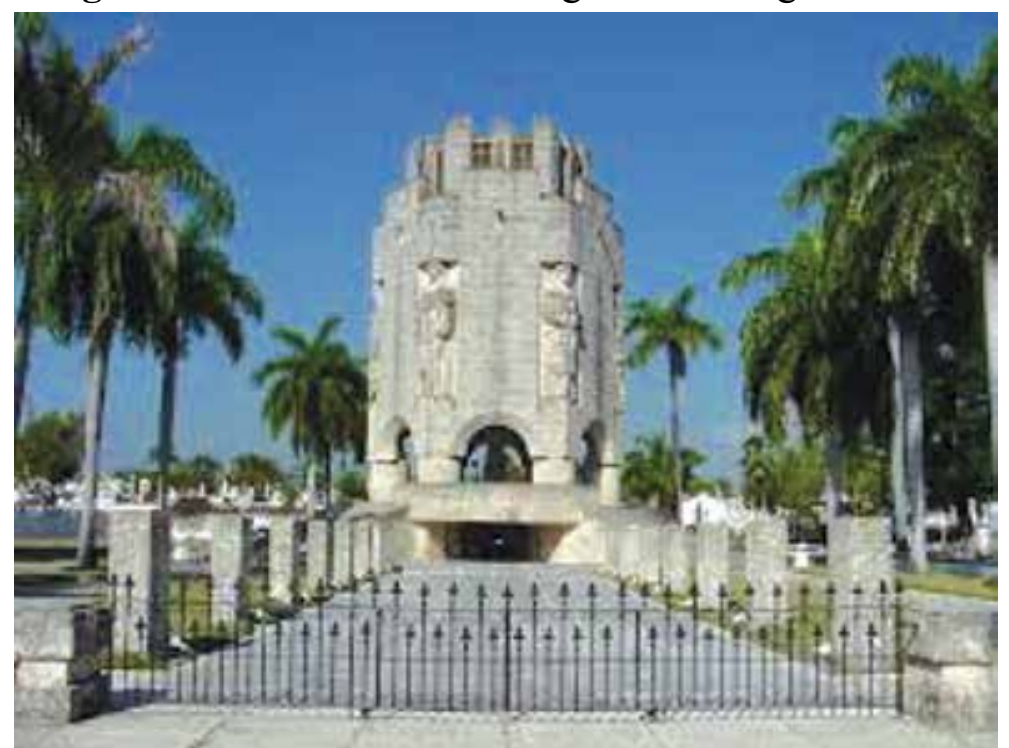

Fuente: Archivo del Grupo Gestor de Patrimonio de la Necrópolis de Colón. 2015.

Figura 2. Cementerio Tomás Acea. Cienfuegos

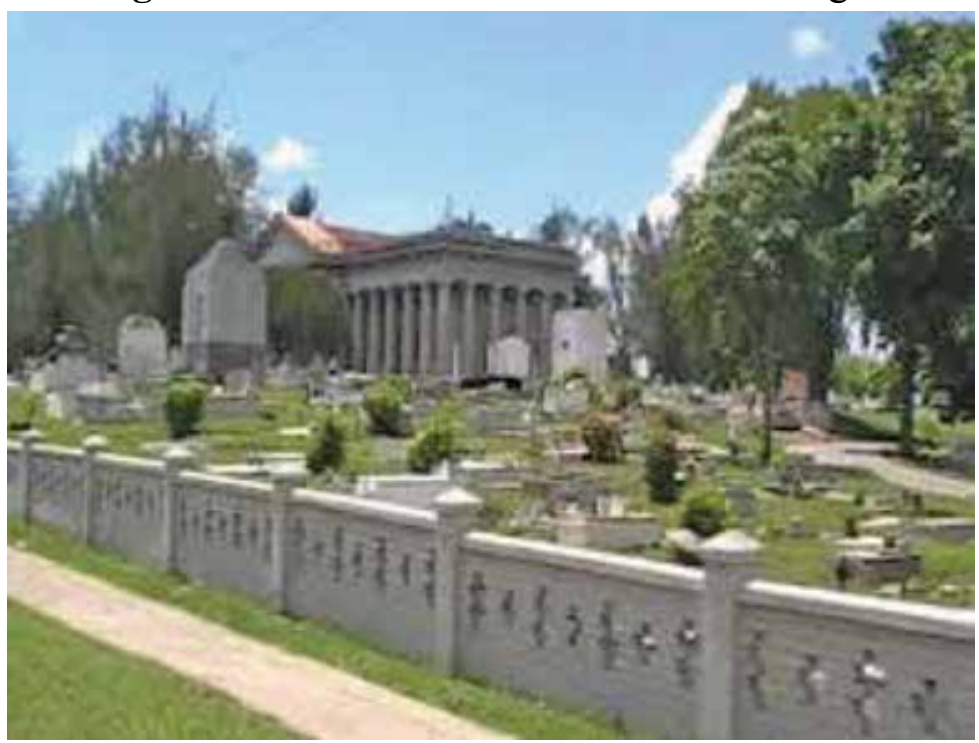

Fuente: Archivo del Grupo Gestor de Patrimonio de la Necrópolis de Colón. 2015. 
La construcción de la Necrópolis de Cristóbal Colón fue concluida el 2 de julio de 1886, los trabajos se prolongaron durante quince años. Es el mayor y más importante del país. El arquitecto español Calixto Aureliano de Loira y Cardoso, concibió el proyecto, que abarcó 56 hectáreas de terreno en forma de rectángulo, de 830 por 630 metros, con cuarteles que están trazados a partir de sendas o calles en forma de cruz, de norte a sur y de este a oeste (Aruca y Salinas, op.cit.).

Figura 3. Portada del Cementerio de Colón. La Habana

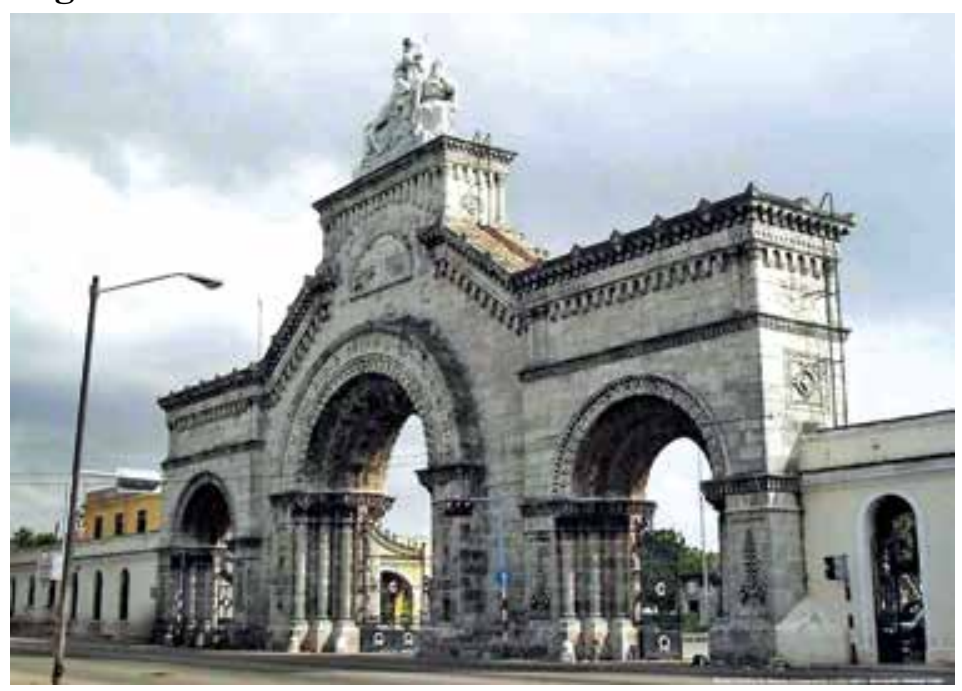

Fuente: Archivo del Grupo Gestor de Patrimonio de la Necrópolis de Colón. 2015. 
Eros Salinas-Chávez, Jeisy Diaz-Fernández. Tourism at the cemeteries. Walking among the dead

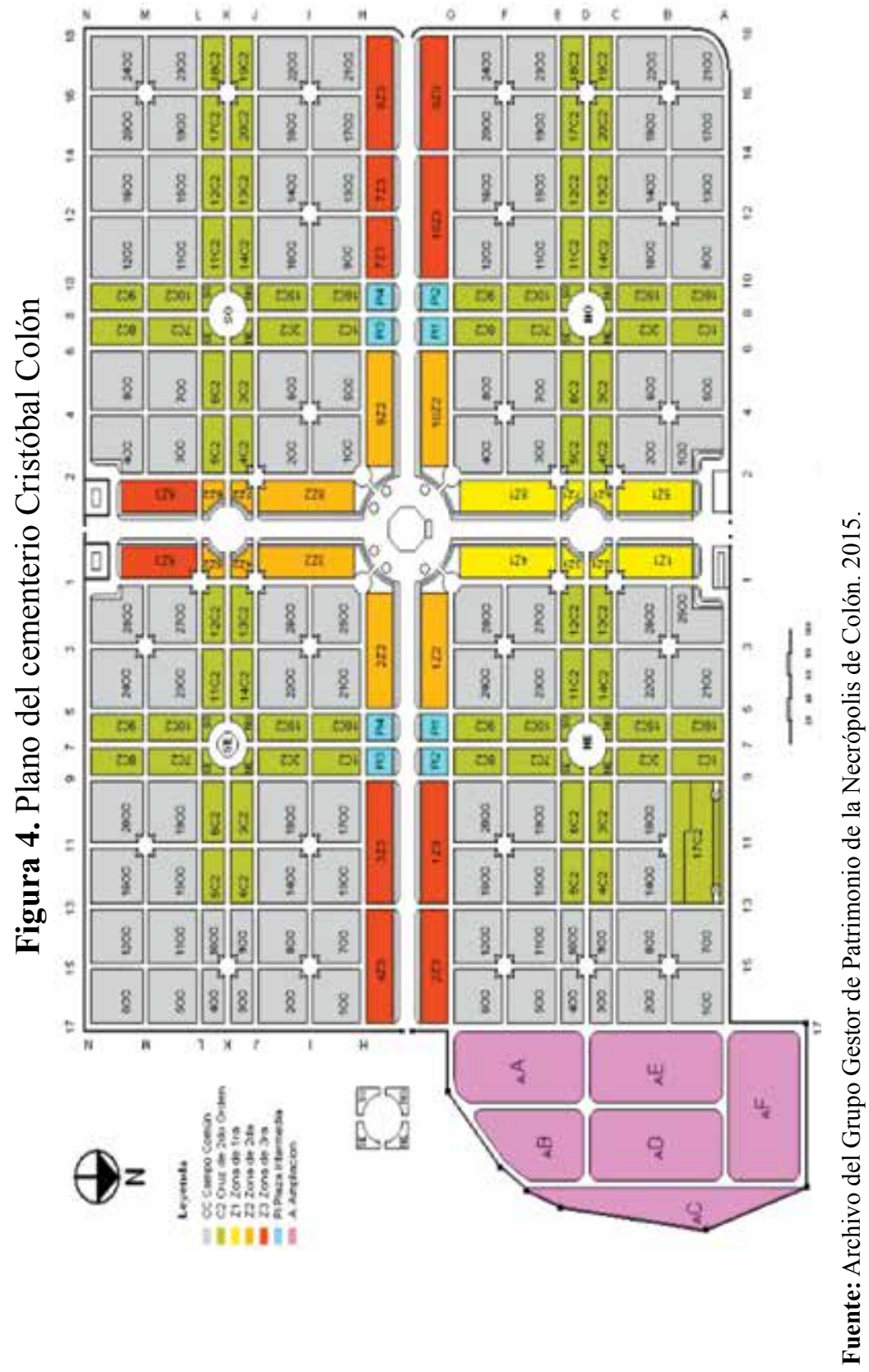


El gran arco triunfal, de tres cuerpos, que constituye el acceso principal a la necrópolis, es una obra monumental de estilo romántico realizada en piedra de cantería tallada con símbolos que aluden a la religión católica y fue adornado, en 1900, por un conjunto de figuras esculpidas en mármol blanco de Carrara, que representan las tres virtudes teologales: fe, esperanza y caridad, de la mano del escultor cubano José Villalta Saavedra. Guardado por tres kilómetros de muros con verjas y copas mortuorias de hierro forjado, posee obras de arte de valor incalculable, donde se distinguen códigos formales diversos como el Neogótico y el Neoclásico, el Art-Decó y el Eclecticismo en general. La capilla principal, de forma octagonal y de tres cuerpos concéntricos, es única en Cuba y se halla decorada con pinturas del insigne artista cubano Miguel Melero.

Sus construcciones funerarias se integran en ese bazar de estilos y gustos a partir de la gran cantidad de materiales constructivos utilizados: mármoles finos, piedra labrada, granito y lapislázuli, entre otros. Posee más de 450 capillas funerarias familiares y de instituciones nacionales y extranjeras, algunas de estas últimas con espacios subterráneos, generalmente, decoradas con vitrales que embellecen las edificaciones. Colecciones de esculturas de diversos tamaños, trabajos de herrería y otras obras decorativas están presentes abundantemente en los panteones, donde sobresalen los epitafios, reflexivos unos, poéticos y satíricos otros, por todos estos valores patrimoniales, la Necrópolis de Colón fue declarada Monumento Nacional en 1987 (Ibid.).

En este cementerio, se cometieron algunos hechos vandálicos en los años ochentas y noventas, de sustracción de distintos elementos artísticos y decorativos en diferentes mausoleos y panteones, lo que motivó a las autoridades a la necesidad de establecer una custodia permanente para proteger este valioso patrimonio al aire libre, y situar a un personal especializado en la custodia en las puertas de salida y en las noches.

A partir del primer decenio de este siglo, ha sufrido un amplio proceso de restauración, que ha abarcado la portada; la capilla, en particular las pinturas de su interior; y todos los monumentos funerarios existentes en la calzada principal, por personal especializado de la Oficina del Historiador de La Habana, que ha devuelto la blancura perdida a los mármoles de Carrara y la brillantez de los bronces. 


\section{Diseño y planificación de productos turísticos cementeriales}

El turismo en los cementerios, como cualquier otro desarrollo, debe ser planificado de manera adecuada. Corresponde esta responsabilidad compartida a los organismos que dirigen la actividad turística, en particular las agencias receptivas; las autoridades públicas que administran y gestionan estos espacios e instalaciones necrológicas; y aquellas que tienen como tarea velar por la conservación del patrimonio. Se considera que solamente a través de la integración de los intereses diversos de estas distintas instituciones y organismos se logrará acercarse a la propuesta de un turismo sostenible, y solo así y no de otra forma, se logrará armonizar la paz y el recogimiento característico y necesario de los cementerios con la presencia de grupos de visitantes; para ello, habrá que reducir al máximo las aglomeraciones de personas e impedir, de esta forma, la "polución visual" y los ruidos, sujetos a las regulaciones establecidas y su correcta aplicación.

A su vez, la gestión del patrimonio, en la actualidad cubana, está en manos de especialistas, funcionarios y profesionales de muy distinta condición y formación, quienes trabajan, de igual manera en distintos organismos e instituciones, con diferentes objetivos y con modos de acción propios. Todos ellos se agrupan en tres grandes ámbitos; a saber, el de la administración pública (organismos gubernamentales nacionales, provinciales y municipales); el académico y profesional (universidades, instituciones académicas y organizaciones de profesionales) y el de la iniciativa privada (Fernández, 2015).

En los cementerios se debe planificar el posible uso turístico, con el propósito de evitar el conflicto entre quienes visitan las tumbas de familiares y amigos, y la presencia de otras personas que ahí llegan con fines de ocio y conocimiento, los cuales desean recibir vivencias y experiencias. En ningún caso, puede el turismo entrar en competencia ni obstaculizar la función social prioritaria que tienen estos lugares y los objetivos higiénico-sanitarios y religiosos que les dieron origen, como tampoco, propiciar la degradación de los recursos histórico-culturales allí existentes.

Para evitar cualquier impacto negativo del uso turístico de estos sitios, se proponen estudiar tres aspectos principales: 
- Capacidad de carta turística diaria de los cementerios de recibir visitantes.

- $\quad$ Diseño de los recorridos de los visitantes.

- $\quad$ Formación del personal, en particular los guías especializados.

Asimismo, se propone, como procedimiento, evaluar la capacidad de carga turística de los cementerios con el empleo de cinco variables, las cuales fueron determinadas por los autores en los numerosos recorridos realizados, y consensuadas en las entrevistas y consultas realizadas a los especialistas:

- $\quad$ La diversidad y riqueza de los recursos patrimoniales allí existentes.

- $\quad$ El área ocupada por estos recursos y su distribución espacial dentro del cementerio.

- $\quad$ El tamaño de los grupos de visitantes.

- $\quad$ El tiempo de duración de las visitas y

- $\quad$ Las posibilidades de gestión y de guías disponibles de las autoridades administrativas de los cementerios.

Estas variables fueron confrontadas con los informantes claves (ver anexo 1); esta herramienta de investigación y los resultados obtenidos, permitieron recomendar criterios de explotación en cuanto a selección y trazado de nuevos recorridos turísticos; tamaño de los grupos de visitantes; y horarios de visitas, entre otros aspectos.

La selección y diseño de los recorridos debe realizarse por un personal especializado del propio cementerio y deberá tomar en cuenta las particularidades de los atractivos turísticos del sitio, en particular, su distribución espacial. Hay cementerios donde se concentran, relativamente en poco espacio, los principales atractivos y existen otros casos en que la distribución de los mismos es mayor y es necesario caminar más para visitarlos.

Atendiendo a los resultados de los expertos consultados, el tamaño de los grupos no debe exceder a doce visitantes por guía especializado, para facilitar la explicación y la comprensión por parte de los visitantes. Debe recordarse que el trabajo del guía se realiza al aire libre y no tiene posibilidades de gritar o alzar la voz, pues sería de muy mal gusto, en un sitio donde predomina y se consume silencio.

Revista Geográfica de América Central Nº Especial ISSN 1011-484X, I Semestre 2017, pp. 243-263 
La cantidad de grupos admisibles, a un mismo tiempo, debe fluctuar entre tres y cuatro, en cementerios con las características y tamaño del de Cristóbal Colón (56 hectáreas). En la actualidad, la llegada concurrente de varios ómnibus en el horario de la mañana dificulta los servicios de los guías especializados, los cuales se ven sobrepasados por el arribo de un gran número de turistas, lo que provoca la necesidad de organizar los servicios en grupos de entre quince y veinte visitantes, lo cual conspira contra la satisfacción de los clientes.

El mejor horario para los recorridos es en la mañana, entre las 9 y las 11:30 a.m, para evitar el fuerte sol del mediodía y reanudar las visitas, de ser necesario, entre las 3 y las 4:30 p.m; los cementerios cierran a las 5:00 p.m, generalmente, por lo cual la visita debe concluir media hora antes de ese momento. La duración de los recorridos guiados puede variar entre media y una hora, se propone una duración promedio de 45 minutos, tiempo suficiente para no aburrir ni abrumar con información excesiva a los visitantes, ni cansarlos con una caminata demasiado larga; un recorrido a pie, entre 1 a $1.5 \mathrm{~km}$, es más que suficiente en este cementerio.

Las posibilidades de gestión que posean las administraciones de los cementerios son importantes a la hora de determinar la capacidad de carga, pues no sólo es necesario contar con valiosos recursos patrimoniales, sino tener la capacidad, disposición y conocimientos para gestionar los mismos, con fines turísticos, en ello juega un papel destacado el número y la preparación de los guías especializados con que se cuente. No sólo en estos productos turísticos, tan particulares, sino en cualquier otro producto turístico, un buen guía puede salvar un recorrido no muy interesante $\mathrm{y}$, viceversa, un mal guía puede echar a perder el mejor recorrido.

\section{Recorridos principales existentes en la Necrópolis de Colón}

- Recorrido general histórico. Tiene como objetivo identificar los valores históricos de la necrópolis, reconocidos en su declaratoria como Monumento Nacional (Resolución 51/87); en el mismo se enlazan personalidades históricas, acontecimientos asociados con ellas o con los movimientos en cuestión (Fernández, op.cit.).

- $\quad$ Recorrido especializado de arquitectura. Pretende transmitir el valor arquitectónico que posee la Necrópolis de Colón, a partir de los diferentes estilos en sus mausoleos y monumentos funerarios. Se 
presentan las construcciones más voluminosos, espléndidas y significativas del cementerio; tanto por su disposición arquitectónica como por su ornamentación artística.

- Recorrido ilustrativo de las esculturas. Constituye una propuesta atractiva para el visitante. Tiene como objetivo transmitir el valor de los elementos esculturales que posee la Necrópolis de Colón materializadas, principalmente, en mármoles italianos y cubanos; comienza por el conjunto escultórico de la puerta principal, el cual representa las tres virtudes teologales, "Fe, Esperanza y Caridad".

- $\quad$ Recorrido especializado sobre curiosidades, mitos y leyendas. Es uno de los más populares dentro de la oferta turística, y tiene como objetivo mostrar la necrópolis como un museo a cielo abierto que atesora numerosos mitos, curiosidades, cultos religiosos y populares, personalidades y sucesos históricos (Aloy, op.cit.).

Figuras 5 a 8. Diferentes vistas de la Necrópolis de Colón, La Habana
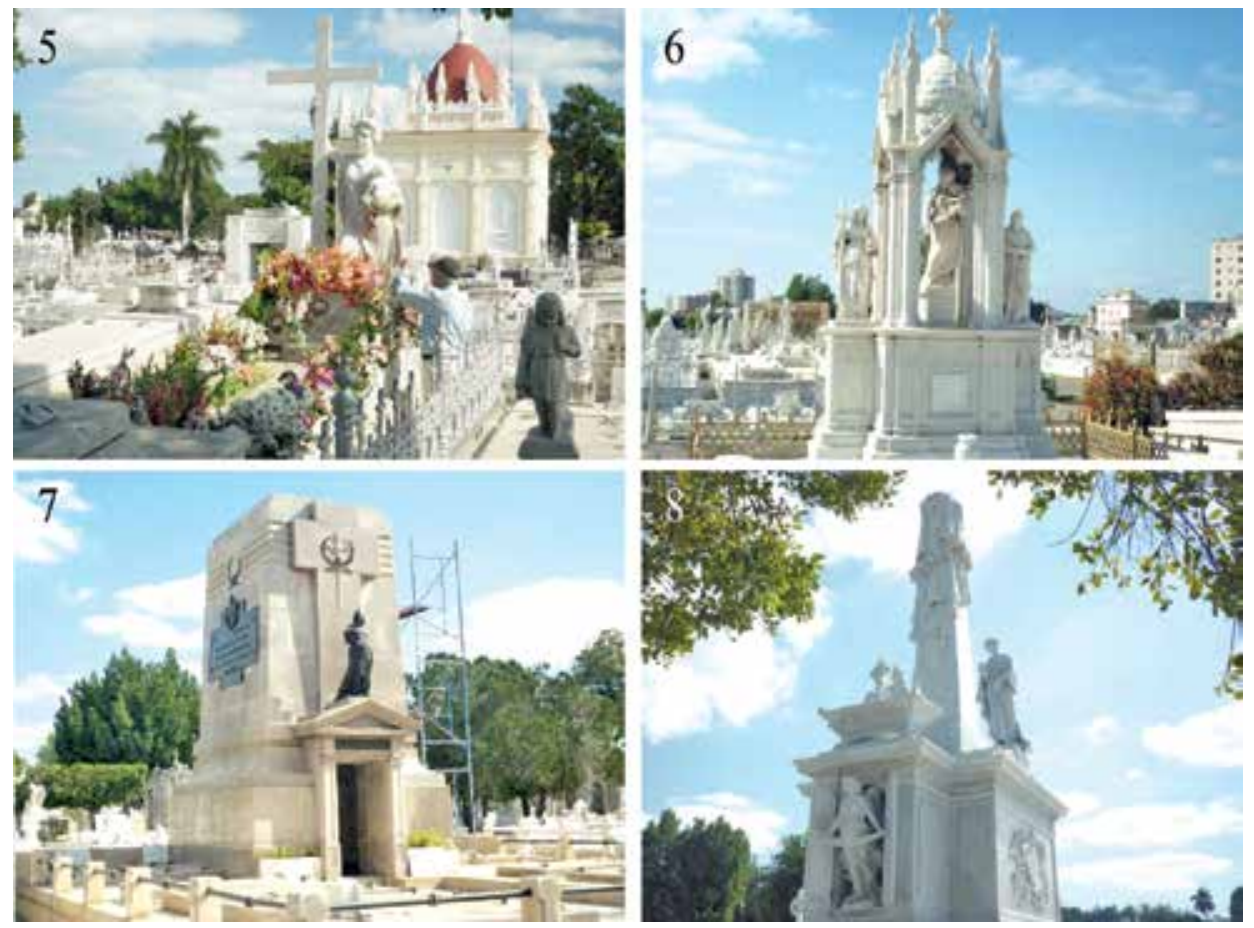

Fuente: Fotos de Eros Salinas Chávez. 2013. 
En tal sentido, se reafirma que la creación de un escenario apropiado en el turismo de patrimonio cultural requiere del análisis y la visión relacionados con la interpretación de éste, a partir del producto turístico que, lógicamente, debe responder al origen y acercamiento más auténtico de la cultura que se trate. Es importante señalar que la interpretación del patrimonio brinda beneficiosos aportes al desarrollo turístico cultural, ya que enriquece el conocimiento dentro de la comunidad a la cual se pertenece y revela significados; valora el patrimonio y despierta la necesidad de su conservación para la posteridad; contribuye a evitar el deterioro del patrimonio, tanto en el ámbito local como global y, de esta manera, estimula actitudes positivas entre los visitantes, y a la población local infunde un comportamiento más acorde con sus valores (Fernández, op.cit.).

Además, la interpretación patrimonial concreta, dentro de su misión, la preservación de importantes recursos económicos para la sociedad y crea una fuente de ingresos y un entorno socioeconómico beneficioso para la comunidad. Asimismo, favorece un buen uso del espacio porque concentra a los visitantes en lugares apropiados, evita sitios vulnerables y elige otros más resistentes (Manzanarez, 2012).

Las visitas de grupos de turistas a los cementerios suelen formar parte de los llamados recorridos de ciudad, que se ofertan como excursiones independientes (opcionales) o como parte de los programas o paquetes turísticos; en todo caso, por ser éste un recorrido al aire libre, se deben tomar en cuenta las condiciones del estado del tiempo en la planificación de los recorridos para evitar, así, las horas de mayor insolación y las posibles precipitaciones.

Las instalaciones de servicios, sean éstas permanentes o temporales, como kiosco su otras ubicadas dentro o en el entorno de los cementerios, son un complemento muy importante para los visitantes y para las funciones del propio cementerio, por ejemplo la venta de flores. Un papel destacado lo tienen la existencia de áreas de parqueo externas a los espacios funerarios, donde puedan estacionarse los vehículos, en particular los ómnibus que trasladan a los grupos de turistas, los cuales no es conveniente se estacionen dentro del propio cementerio por los ruidos ocasionados y por la imagen de ocio que ellos transmiten, para nada vinculada con el reposo y paz propio de los cementerios.

Algunos cementerios tienen una o dos salas, generalmente en el área de la entrada, que sirven como museos, centros de interpretación y áreas 
de coordinación de las visitas; en estos espacios, por lo común cerrados, se puede exhibir una muestra de elementos funerarios museables significativos del lugar, y ofertarse, además, mapas y guías turísticas del cementerio, postales y otros objetos.

Cuadro 1. Capacidad de carga actual del Cementerio de Colón, La Habana, Cuba

\begin{tabular}{|l|l|l|l|c|}
\hline \multicolumn{1}{|c|}{ Cementerio } & $\begin{array}{l}\text { Número total de } \\
\text { grupos de visitantes } \\
\text { organizados, por día }\end{array}$ & $\begin{array}{l}\text { Número máximo } \\
\text { de visitantes, por } \\
\text { día }\end{array}$ & $\begin{array}{l}\text { Número máximo } \\
\text { de visitantes, } \\
\text { por semana } \\
\text { (considerar 6 días) }\end{array}$ & $\begin{array}{c}\text { Total } \\
\text { anual }\end{array}$ \\
\hline $\begin{array}{l}\text { Cristóbal Colón } \\
\text { (La Habana) }\end{array}$ & $\begin{array}{l}\text { 12 por la mañana y } 4 \\
\text { por la tarde. } \\
\text { De } 16 \text { a } 12 \text { grupos } \\
\text { diarios. }\end{array}$ & $\begin{array}{l}\text { De 264 a } 320 \\
\text { turistas. }\end{array}$ & $\begin{array}{l}\text { De 1584 a 1920 } \\
\text { turistas. }\end{array}$ & $\begin{array}{l}\text { De } 82000 \\
\text { a } 100000 .\end{array}$ \\
\hline
\end{tabular}

Fuente: Estimaciones actuales de los autores sobre la base de los indicadores señalados, las visitas realizadas y las opiniones de los expertos consultados.

Las cantidades de visitantes que aparecen en el cuadro 1 proporcionan un margen de protección para tratar de evitar cualquier impacto negativo sobre los recursos histórico-culturales y arquitectónicos presentes, sobre las costumbres de uso y ritos de la población residente y sobre los servicios funerarios que los cementerios brindan.

\section{Visitantes recibidos en el Cementerio de Colón}

En 1990, el Cementerio de Colón recibió 54946 turistas foráneos al formar parte de diferentes recorridos programados por la ciudad de La Habana, con un promedio mensual de 4580 visitantes organizados en grupos (Salinas y Blanco, 1991); la tendencia al incremento se mantuvo y, en 1991, se recibieron un total anual de 59256 turistas organizados; no se cuentan en estas cifras aquellos que se mueven individualmente (Aruca y Salinas, op.cit.). El número de visitantes ha ido creciendo paulatinamente, con altas y bajas. Una continuación de esta investigación, realizada diecinueve años después, reportó que, en 2009, se recibieron 75643 visitantes extranjeros; en esta información no se contabilizan los visitantes nacionales pues, hasta la fecha, quienes llegan por motivos turísticos son mínimos. 
Figura 9. Visitantes recibidos en la Necrópolis de Colón, 2009-2014.

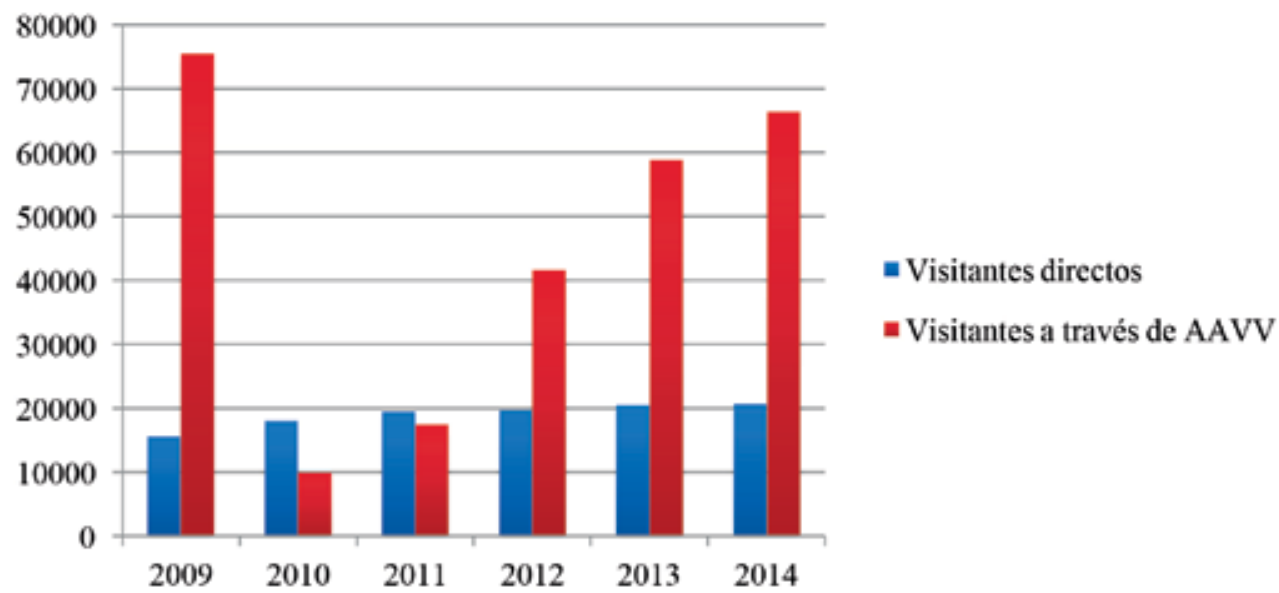

Fuente: Servicios Necrológicos de la Provincia de La Habana, 2015.

Según los Servicios Necrológicos de la Provincia de La Habana, consultados en 2015, el mayor ingreso de turistas se realiza durante los meses de la temporada alta internacional, de enero a mayo, mientras los meses de menor recepción de visitantes extranjeros son julio y agosto, que corresponden con la temporada baja del turismo internacional.

Existe una notable diferencia, de alrededor del $83 \%$ menos, entre los visitantes recibidos en 2009 (75643) con respecto a 2010 (10037); a nuestro juicio, la causa fundamental de este descenso se originó por el aumento de precio en la entrada, efectuado por la Empresa de Servicios Necrológicos de La Habana en el primer trimestre del 2010, y que significó un alza para los operadores receptivos nacionales de tres a cinco dólares de Estados Unidos. Estos operadores turísticos, en respuesta a esa decisión inconsulta y al considerar excesivo dicho aumento, determinaron, por su parte, excluir de sus tours de ciudad al Cementerio de Colón; en consecuencia, disminuyeron de súbito la recepción de los grupos organizados de visitantes. Aunque estas discrepancias tuvieron, posteriormente, una solución negociada entre las partes, ya el daño al producto turístico estaba hecho, si bien ha habido una recuperación paulatina hasta la actualidad. Esto es un claro ejemplo de la necesaria conjugación de intereses que debe existir entre las distintas instituciones vinculadas con el uso turístico de los cementerios, en cualquier lugar. 
En la figura 10 se aprecia el crecimiento de los ingresos por concepto de las entradas al Cementerio de Cristóbal Colón de los visitantes, tanto de grupos organizados como de turistas individuales. El promedio mensual de ingresos recibidos, por concepto de entrada de grupos, en 2014, fue de 5800 dólares de Estados Unidos y de 8100 dólares de Estados Unidos, promedio mensual, por el turismo individual (Fernández, op.cit.).

Cuadro 10. Necrópolis de Colón: ingresos por concepto de turismo, 2011 a 2013

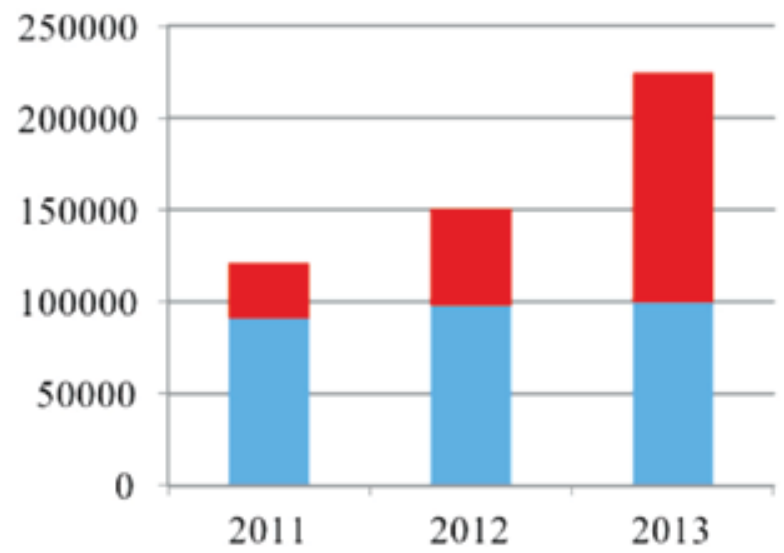

- Ingresos por visitas de turistas a través de AAVV (CUC). - Ingresos por visitas de turistas individuales (CUC)

Fuente: Servicios Necrológicos de la Provincia de La Habana, 2015.

(AAVV. Agencias de viajes. Un CUC equivalía a 1,10 dólares de Estados Unidos en esa fecha)

\section{Conclusiones}

El desarrollo turístico en los cementerios constituye una fuente alternativa de ingresos, creación de nuevos empleos y otros beneficios para la población local y, más importante, contribuye al conocimiento de los visitantes nacionales y foráneos respecto a la historia y cultura de esos lugares.

Una oferta turística cultural, a partir de los cementerios patrimoniales, es parte del turismo de intereses especiales. Si se realiza en forma planificada, ofrece la oportunidad de contribuir al incremento o generación de un criterio conservacionista entre los administradores de los cementerios y los operadores turísticos, con el apoyo de la sociedad. 
Las cantidades de personas recibidas como visitantes en los cementerios, en un cierto espacio y tiempo determinado, deben estar sujetos a un proceso constante de ajuste, que tome en cuenta, entre otros parámetros, el posible impacto negativo sobre los valores patrimoniales allí existentes; la calidad de la experiencia cognoscitiva percibida por los visitantes; la estacionalidad de las visitas y horario de los recorridos; y, finalmente, la capacidad operativa de la propia administración para brindar, por una parte, los servicios de guías especializados a los visitantes y, por otra, los servicios necrológicos que le corresponde brindar a la población local, los cuales no pueden deteriorarse por causa del turismo.

Los cementerios cubanos son desconocidos; ni los ciudadanos, ni los turistas extranjeros conocen el patrimonio histórico, arquitectónico y artístico que albergan estos recintos, por lo que deberá realizarse y ejecutarse un plan de interpretación, que tuviera por objetivo "estimular la educación y sensibilización patrimonial de la población de manera innovadora".

\section{Agradecimientos}

A la Dirección Provincial de Servicios Necrológicos, en particular, a la especialista Dania Álvarez Guevara de esa Dirección; al Grupo Gestor de Museología y Patrimonio de la Necrópolis de Colón; y al guía especializado Orlando Infante de la Agencia Viajes Cubanacán.

Dedicado a la MSc. Teresita Chepe Rodríguez $(\dagger)$. Amiga y entusiasta estudiosa de los cementerios.

\section{Bibliografía}

Aloy, T. (2013). Estudio sobre tradiciones y costumbres funerarias y religiosas en la Necrópolis Cristóbal Colón. Documento del Equipo Técnico de Historia, Conservación e Informática de La Necrópolis de Colón. La Habana.

Aruca, L. y Salinas, Er. (1992). "Turismo en los cementerios. El cementerio Cristóbal Colón”. En: Revista Revolución y Cultura. (6), 24-29. Aulet, S. (2010). Itinerarios culturales en Cataluña: de la idea al proyecto real. En Gali, N. (coord.) Itinerarios culturales. La experiencia del Camino de los Diamantes. Girona: Documenta Universitaria

Benayas, J. y Muñoz, M. (2007). Boletín de interpretación No. 23 Artículo "Evaluación de la interpretación en Parques nacionales (o saber qué 
hacemos, cómo lo hacemos y cómo mejorarlo). Recuperado: http:// www.interpretaciondelpatrimonio.org.

Chepe, T. (1997). Reseña histórica y cultural de la ciudad de Cienfuegos.

Material de apoyo para Curso de Postgrado El patrimonio cienfueguero. Centro Provincial de patrimonio. (Inédito).

Fernández, J. (2015). Propuesta del plan interpretativo para desarrollar productos turísticos culturales de la Necrópolis de Colón. Tesis de Maestría en Gestión Turística. Facultad de Turismo. Cuba.

Grupo Gestor de Patrimonio de la Necrópolis de colón. 2015. Memorias del Segundo Taller de Cementerios y Espacios Funerarios. Memorias. La Habana. (Inéditas).

Iznájar, B. (2013). Los 10 museos más extraños de Europa. En: Revista Viajar, 414, 95-97.

Jaffari, J. (2002). Enciclopedia de Turismo. Editorial Síntesis. Madrid.

Lennon, J. y Foley, M. (2000). Dark Tourism: In the footsteps of death and disaster. London. Cassell.

Manzanarez, P. (2012). Un recorrido por los camposantos más bellos. Recuperado: http://observatorioinversis.com/sin-categoria/ el-turismo-de-cementerios-llega-a-españa/

Martínez Trillo, O. (2014). Aproximación al turismo funerario: análisis de oferta de la Ruta Europea de Cementerios en Cataluña. Maestría en Turismo Cultural. Facultad de Turismo. Universidad de Girona.

Morales, J. (2001). Guía Práctica para la Interpretación del Patrimonio. Consejería de Cultura de la Junta de Andalucía y TRAGSA.

Perello, J. (2005). Selección de Textos sobre Interpretación del Patrimonio. Centro de Estudios Turísticos. Universidad de La Habana.

Red Chilena de Cementerios Patrimoniales. (2006). Carta Internacional de Morelia. Cementerios patrimoniales y arte funerario. Michoacán. En: Revista Javeriana18 (1) 65.

Richards, G. (2006). "Cultural tourism in Europe: recent developments. En: Actas del Congreso Europeo sobre itinerarios Culturales y Rutas Temáticas. (Ed.) Logroño. Fundación Caja Rioja, Madrid.

Salinas, Er. y Blanco, A. (1991). "Turismo en los cementerios". En: Revista Latinoamericana de Turismo, 1 (4), 262-266.

Salinas, Er. (2013). Geografía y Turismo. Aspectos territoriales del manejo y gestión del turismo. Editorial Félix Varela. La Habana. 
Seaton A,V. (2002). Thanatotourism's final frontiers? In: Tourism Recreation Research 27 (2) 73-82.

Stone, P. y Sharpley, R. (2008). Consuming dark tourism: A Thanatological Perspective.Annals of TourismResearch, 35(2), 574-595.

Vento Canosa, E. (2002). La última morada, Ediciones Matanzas, Matanzas.

Recuperado:http://www.paris.fr/es/patrimonio-y-monumentos/cementerios-parisinos/p8238 www.enmemoria.com/cementerios.../cementerio-de-staglieno.html http:/www.minube.com/tag/cementerios-estados.unidos-p242 